\title{
Down-regulation of long non-coding RNA ESCCAL_1 inhibits tumor growth of esophageal squamous cell carcinoma in a xenograft mouse model
}

\author{
Yuanbo Cui ${ }^{1}$, Wei Wu ${ }^{2}$, Pengju Lv ${ }^{1}$, Jianying Zhang ${ }^{3}$, Bingqing Bai ${ }^{4}$ and Wei Cao ${ }^{1}$ \\ ${ }^{1}$ Translational Medicine Center, Zhengzhou Central Hospital Affiliated to Zhengzhou University, Zhengzhou, People's Republic \\ of China \\ ${ }^{2}$ Helen Dillar Family Cancer Center, Department of Medicine, University of California in San Francisco, San Francisco, CA, USA \\ ${ }^{3}$ Henan Academy of Medical and Pharmaceutical Sciences, Zhengzhou, People's Republic of China \\ ${ }^{4}$ Department of Clinical Medicine, Zhengzhou University, Zhengzhou, People's Republic of China \\ Correspondence to: Wei Cao, email: caoweiyu@hotmail.com
}

Keywords: esophageal squamous cell carcinoma; long non-coding RNA; ESCCAL_l; tumor growth; phospho-kinase

Received: August 31, $2017 \quad$ Accepted: November 15, $2017 \quad$ Published: December 11, 2017

Copyright: Cui et al. This is an open-access article distributed under the terms of the Creative Commons Attribution License 3.0 (CC BY 3.0), which permits unrestricted use, distribution, and reproduction in any medium, provided the original author and source are credited.

\section{ABSTRACT}

Esophageal squamous cell carcinoma (ESCC) is one of the most lethal malignant cancers with high incidence and mortality. Current reliable effective diagnostic and prognostic biomarkers are very limited in clinic. Emerging evidence indicates that dysregulated expression of the long non-coding RNAs (IncRNAs) was examined in various types of cancer including ESCC. ESCC associated IncRNA_1 (ESCCAL_1) was first time identified to be increased expression in ESCC, and therefore named by our research team. However, its potential function in the progression of ESCC remains unclear. In this study, we investigated the effect of ESCCAL_1 knockdown on ESCC tumorigenicity using a xenograft mouse model and explored the underlying molecular mechanism. Here we showed that ESCCAL_1 knockdown significantly inhibited EC9706 cell growth in nude mice. Interestingly, we also found that reduced expression of ESCCAL_1 resulted in distinct alterations of relative phosphorylation level of kinases (p-p38a, p-JNK, p-FAK and p-Src), and significant changes of the expression level of apoptosis-related proteins (p53, BAX, Bcl-2 and Caspase-3). In summary, our results suggest that InCRNA ESCCAL_1 is a potential diagnostic and prognostic target of ESCC.

\section{INTRODUCTION}

Esophageal squamous cell carcinoma (ESCC) is one of the most malignant cancers worldwide, and the predominant histologic type of esophageal carcinoma in Asia [1]. ESCC is the sixth leading cause of cancer-related death with more than 400,000 deaths each year [2-3]. There is obvious regional distinction in ESCC incidence, which mainly occurs in the "esophageal cancer belt" from northeast China such as Henan, Shanxi and Hebei provinces to the Middle East [2-3]. Despite the development of treatment for ESCC including chemotherapy, radiation therapy, surgery and preventive approaches, the majority of patients are diagnosed as ESCC at the advanced stage [4-5].
Five-year overall survival rate of ESCC remains less than $30 \%$ due to recurrence, drug resistance and metastasis [6-7]. Therefore, to explore novel effective biomarker and diagnostic and prognostic target for ESCC is an unmet need.

Long non-coding RNAs (lncRNAs) are new members of non-coding RNAs in the mammalian genome, they are over 200 nucleotides in length. In general, lncRNAs have no protein-coding capacity and lack a complete open reading frame (ORF) [8]. Although the transcripts of lncRNAs do not translate into proteins, lncRNAs play crucial roles in a variety of biological and physiological processes in regulating chromatin modification and gene expression profiling [9]. In recent years, IncRNAs have been reported to function as oncogenes or competing 
endogenous RNA (ceRNA) involving in proliferation, apoptosis, invasion and metastasis in many cancers [10-12]. To date, only a few functional lncRNAs in ESCC have been identified and the underlying mechanisms are not fully elucidated [13-15]. Esophageal squamous cell carcinoma associated long non-coding RNA transcript 1 (ESCCAL_1) was first identified in 2013 and named by our team after validation study [16]. We have previously demonstrated that expression of IncRNA ESCCAL_1 was significantly increased in ESCC and inhibition of ESCCAL_1 expression promotes apoptosis and decreasing invasion in ESCC cell lines [17]. However, the detailed molecular mechanisms of ESCCAL_1 on ESCC tumor growth in vivo remains poorly understood.

In present study, we evaluated the effect of ESCCAL_1 knockdown on ESCC tumorigenicity in a xenograft mouse model, and further investigated signaling pathways alterations in xenografted tumor samples using protein arrays.

\section{RESULTS}

\section{Effect of ESCCLA_1 knockdown on ESCC tumorigenesis in vivo}

In order to investigate the effect of ESCCAL_1 knockdown on tumor growth, nude mice were injected subcutaneously with either EC9706 cells expressing ESCCAL_1 negative control (NC-EC9706) or EC9706 cells expressing ESCCAL_1-RNAi knockdown (ESCCAL_1-KD EC9706). Each group contains 6 mice. The tumor volume in KD group showed significantly reduced $(p<0.005)$ compared to the mice in NC group at six different time points [day $13(p=0.001)$, day 15 $(p=0.004)$, day $17(p<0.001)$, day $19(p<0.001)$, day $21(p<0.001)$ and day $23(p<0.001)]$ (Figure 1A). The tumor weight in KD group also displayed dramatically decreased compared to the mice in NC group on $23 \mathrm{~d}$ $(p<0.001)$ (Figure 1B). These results demonstrated that over-expression of ESCCAL-1 promotes tumor growth and down-regulation of ESCCAL_1 inhibits ESCC tumor growth in vivo.

\section{Identification of protein signaling pathways in ESCCAL_1-driven tumor growth}

The process of tumorigenesis is complex and may involve in many signaling pathways associated with cell proliferation and survival. Given the complexity and possible cross-talk among these signaling pathways, a human phospho-kinase array was used to screen ESCCAL_1-mediated key signaling pathways during tumor formation in xenograft model. The total proteins from xenografted tumor samples of sacrificed mice (day 23) were subject to kinase protein phosphorylation detection on 45 protein array. The differential expression levels of kinase proteins between tumor samples from two groups were quantified for comparison. Our result showed that the relative phosphorylation levels of several kinases were increased or decreased between the two groups (Figure 2A). When compared with the NC group, the proteins levels of phosphorylated p38 $\alpha$ (p-p38 $\alpha$ ) and phosphorylated signal transducer and activator of transcription 5a (p-STAT5a) in the KD group were elevated. We also observed that the proteins levels of phosphorylated c-Jun N-terminal kinase ( $\mathrm{p}$-JNK), phosphorylated focal adhesion kinase (p-FAK), phosphorylated glycogen synthase kinase $3 \beta$ (p-GSK3 $\beta$ ) and phosphorylated
A

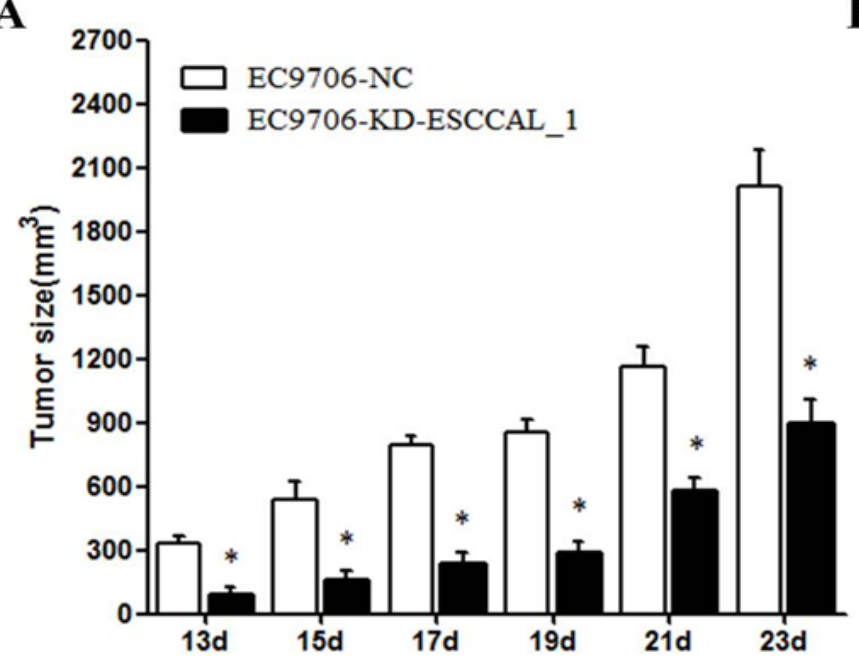

B

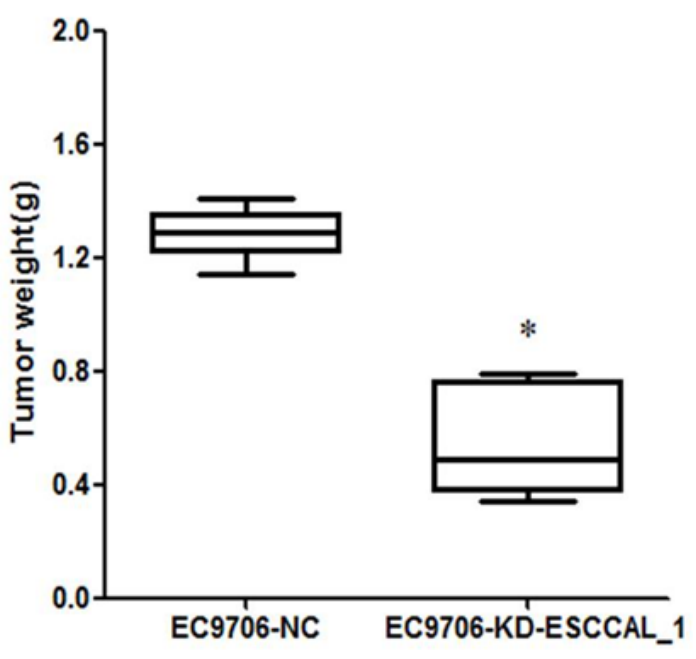

Figure 1: Effect of ESCCAL_1 knockdown on EC9706 cell growth in nude mice. (A) Nude mice were subcutaneously injected with either mock siRNA expressed EC9706 cells (EC9706-NC) or ESCCAL_1 shRNA expressed EC9706 cells (EC9706-KDESCCAL-1). $N=6$ for each group. Tumor volume was measured post-injection on $13 \mathrm{~d}, 15 \mathrm{~d}, 17 \mathrm{~d}, 19 \mathrm{~d}, 21 \mathrm{~d}$ and $23 \mathrm{~d}$. (B) Tumor weight of xenografts tumors from sacrificed mice in two groups on day 23 was measured. Data are presented as mean \pm SEM. ${ }^{*} p<0.05$ is significant difference when compared KD-ESCCAL-1 with NC group of mice. 
sarcoma ( $\mathrm{p}-\mathrm{Src}$ ) were decreased in slowly growing tumor tissues following ESCCAL_1 knockdown (Figure 2A-2B). These data suggest that ESCCAL_1 knockdown predominantly decreased activities of protein kinases.

\section{ESCCLA_1 knockdown decreased phosphorylation of JNK1, FAK and Src}

We further verified this differential expression of kinase proteins in tumors derived from xenografts using conventional Western blot analysis. As shown in Figure 3, the proteins levels of total JNK1 (t-JNK1), total FAK (t-FAK) and total Src (t-Src) had not significant difference between ESCCAL_1-KD group and NC group. However, the expression levels of p-JNK1, p-FAK and p-Src were reduced significantly in the tumors after ESCCAL_1 knockdown when compared to the NC group $(p<0.001)$ (Figure 3A-3B). While Western blot results did not detect significant change of $\mathrm{p}-\mathrm{STAT} 5 \mathrm{a}$ and $\mathrm{p}-\mathrm{GSK} 3 \beta$ expression in the tumor tissues from KD group when compared to NC group $(p>0.001)$ (Figure 3A-3B). Put them together, over-expression of ESCCAL 1 promotes tumor growth through activation of p-JNK1, p-FAK and p-Src-mediated signaling pathways.

\section{Over-expression of ESCCAL_1 activates survival pathway and decrease apoptosis}

In order to examine the effects of ESCCAL_1 knockdown on apoptotic signaling pathways, Western blot analysis was used to detect gene expressions invovling apoptosis and cell survival including p-p38 $\alpha$, p53, p21, Bcl-2, BAX and Caspase-3. Our data revealed that ESCCAL_1 knockdown significantly increased the phosphorylation of $\mathrm{p} 38 \alpha$, which was consistent with the result of phosphor-kinase array $(p<0.05)$ (Figure 4A-4B). We also noted that down-regulation of ESCCAL_1 could dramatically decrease the anti-apoptotic protein $\mathrm{Bcl}-2$ and increase the pro-apoptotic proteins $\mathrm{p} 53, \mathrm{BAX}$ and Caspase-3 $(p<0.05)$ (Figure 4A-4D), but had no effect on $\mathrm{p} 21$ (data not shown). These data indicated that inhibition of ESCCAL_1 expression increased apoptosis and decreased survival in tumors.

\section{DISCUSSION}

ESCCAL_1 is a novel lncRNA with oncogenic potential that was originally identified in ESCC using lncRNA array and bioinformatic analysis by our team [16]. Previous studies have shown that knockdown of ESCCAL_1 by small interfering RNAs could inhibit invasion and promote apoptosis in ESCC cell line EC9706 [17], high expression of ESCCAL 1 transcript was found to be correlated with poor prognosis of ESCC [18]. However, the specific role of ESCCAL_1 in ESCC tumorigenesis remains to be elucidated. In this study, we observed that knockdown of ESCCAL 1 could remarkably inhibit tumor growth of ESCC in a xenograft mouse model.

Consistent with our previous study that knockdown of ESCCAL_1 suppresses ESCC cell proliferation in vitro (data not shown), our result of tumor growth assay in vivo also revealed that down-regulation of ESCCAL_1 inhibits ESCC cell growth in xenografts nude mice. The phosphorylation status of kinase proteins directly reflects their activities, these activated proteins represent core cellular pathways including proliferation, differentiation, senescence and apoptosis [19]. So we screened multiple signaling pathways which might be
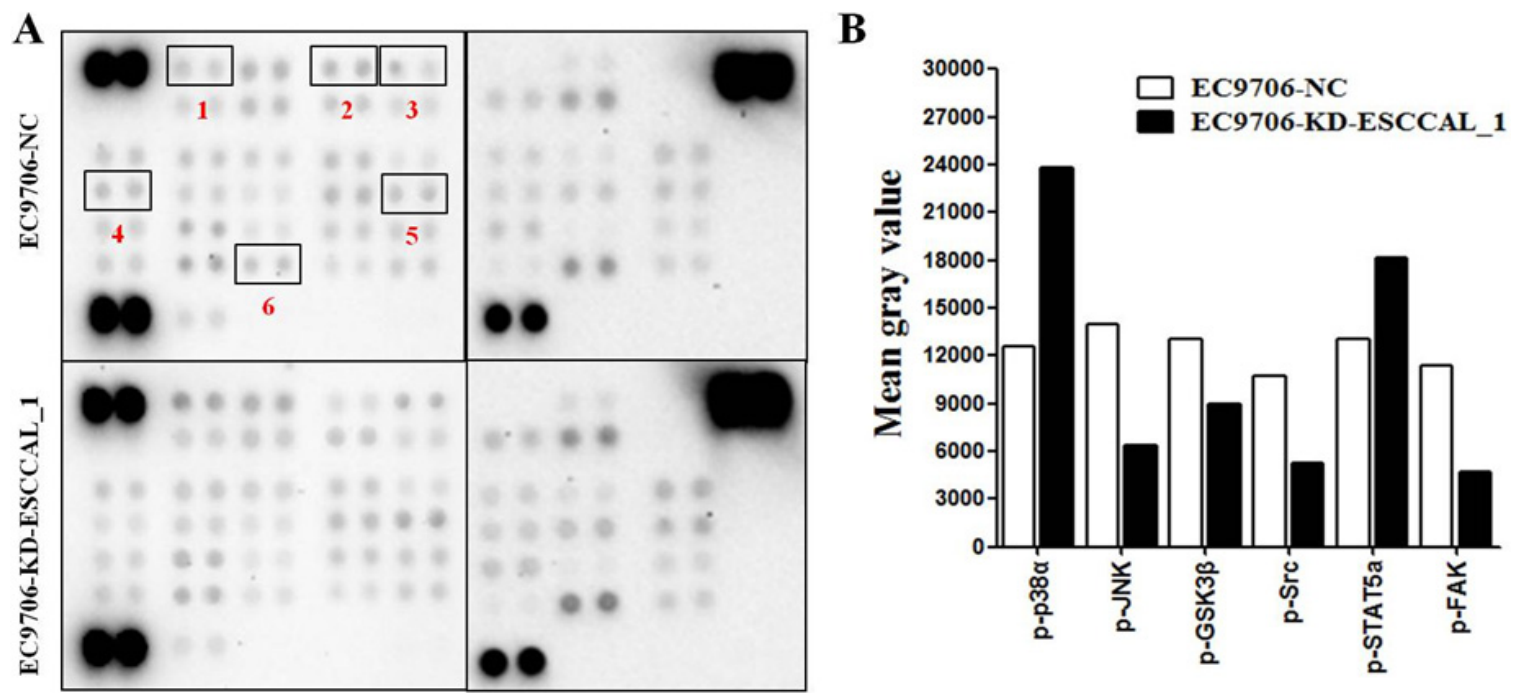

Figure 2: Screening of the phosphorylation levels of protein kinases in tumor tissues. (A) Detection of phosphorylated proteins by using the phospho-kinase membrane array, the differential expression of six kinase proteins was highlighted with red box (1.p-p38a, 2.p-JNK, 3.p-GSK3ß, 4.p-Src, 5.p-STAT5a, 6.p-FAK). (B) Quantification by mean gray value revealed the changes of relative phosphorylation levels of kinases in ESCCAL_1 KD group relative to NC group. 
affected by ESCCAL_1 knockdown in xenografts tumor tissues using phospho-kinase array. We found a series of phosphorylation of kinases proteins whose expression levels were either increased or decreased following ESCCAL 1 knockdown, these kinase proteins include p-p38 $\alpha$, p-JNK, p-GSK3 $\beta$, p-Src, p-STAT5a and p-FAK. The phosphorylation levels of these kinases were verified independently by Western blot.

Previous study reported that up-regulation of GSK-3 $\beta$ contributed to an aggressive phenotype and cell proliferation in ESCC [20]. The STAT5a is the major isoform of STAT5 except STAT5b. It has been shown that both of the STAT5 isoforms were involved in many human cancers, and activation of STAT5a was closely related to tumor progression [21]. However, our data of Western blot showed that the phosphorylation of GSK-3 $\beta$ and STAT5a had no significant changes after ESCCAL_1 knockdown, suggesting that down-regulation of ESCCAL_ 1 inhibiting ESCC tumor growth involved in other signaling pathway. It has been reported that Src was aberrantly activated in many cancers and correlated to poor prognosis of nonsmall cell lung cancer and ESCC [22-23]. Increasing studies indicated that Src plays a key role in promoting tumor growth, invasion and metastasis via regulation of many downstream signaling molecules including FAK and JNK, which are widely considered to be crucial for cell proliferation and migration [23-24]. Our results displayed that the phosphorylation levels of Src, FAK and JNK were significantly decreased following ESCCAL_1 knockdown (Figure 5), suggesting that down-regulation of ESCCAL_1 inhibits tumor growth probably by inactivation of $\overline{\mathrm{Src}} /$ FAK/JNK pathway.

The p38 $\alpha$ pathway is known as a negative regulator in cell proliferation and tumorigenesis. Activation of $\mathrm{p} 38 \alpha$ can inhibit tumorigenesis and tumor cell growth, which suggests that $\mathrm{p} 38 \alpha$ is a tumor suppressor [25-26]. Tumor suppressor p53 plays an important role in regulating cell cycle and apoptosis in many cancers via repair of damaged DNA. If DNA repair fails, accumulation of p53 can stimulate the process of apoptosis to clear away the damaged cells. Previous study reported that activation of p38 $\alpha$ induced increase of p53, thus triggered apoptosis and cell cycle arrest [27]. Consistent with this study, we found that down-regulation of ESCCAL_1 resulted in a significant increase of p-p38 $\alpha$ and p53. Our data also showed that apoptosis associated proteins Caspase- 3 and BAX were dramatically increased and Bcl-2 significantly decreased after ESCCAL_1 knockdown. Caspase-3 is a major executioner protein of apoptosis which can be activated by both extrinsic and intrinsic pathways. It has been shown that the p53 promotes apoptosis through interactions with Caspase- 3 and Bcl-2 family proteins including BAX both in vitro and in vivo in recent studies [28-30]. According to our results, it can be speculated
A

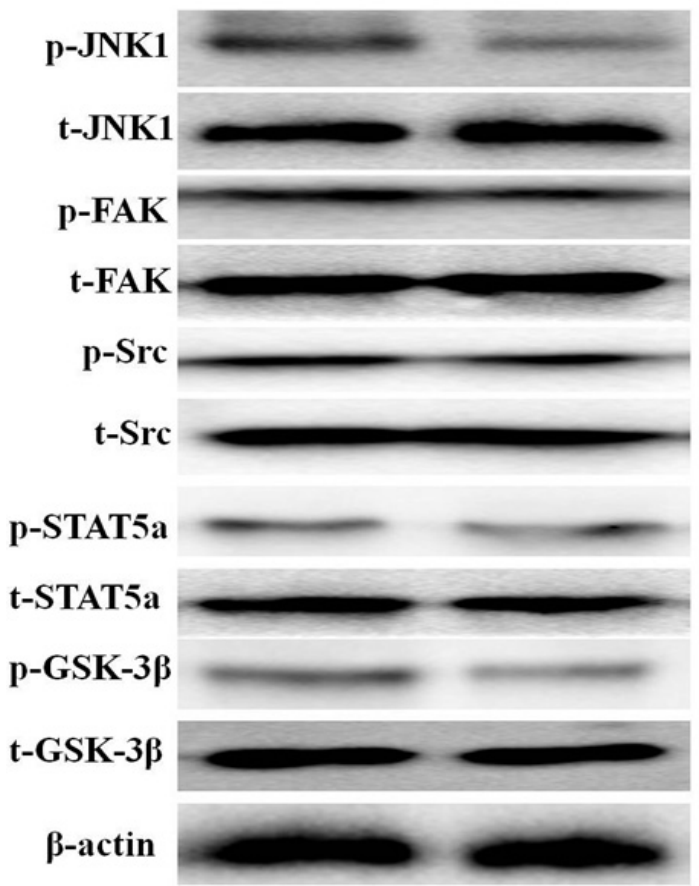

B

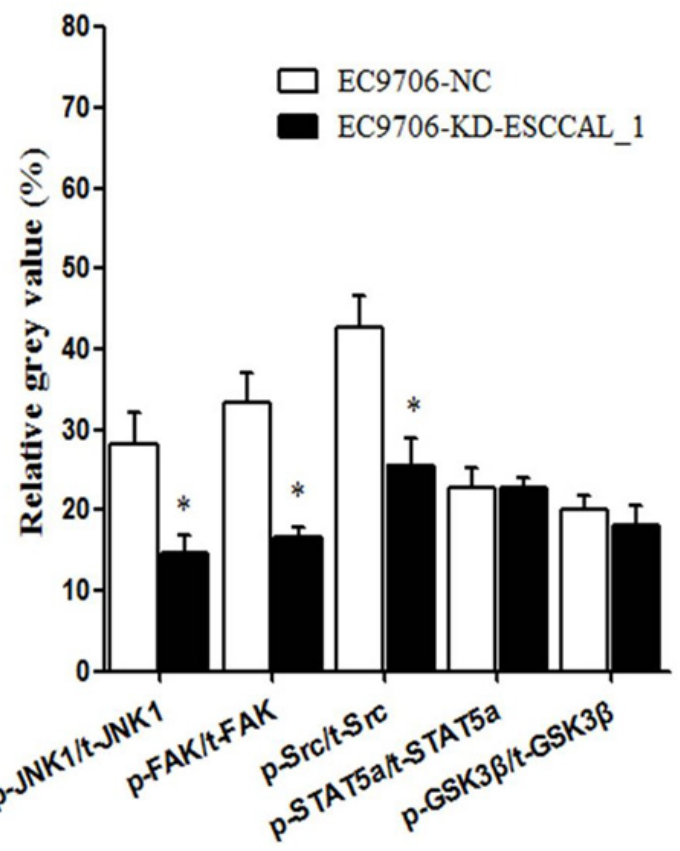

Figure 3: Down-regulation of ESCCAL_1 decreased protein levels of p-JNK1, p-FAK and p-Src confirmed by western blot assay. (A) Total protein lysates from ESCCAL_1 KD group or NC group were subject to Western blot detection with different antibodies [p-JNK1, t-JNK1, p-FAK, t-FAK, p-Src, t-Src, p-STAT5a, total STAT5a (t-STAT5a), p-GSK3 $\beta$ and total GSK3 $\beta$ (t-GSK3 $\beta$ )]. (B) Quantitative protein levels of p-JNK1, p-FAK, p-Src, p-STAT5a and p-GSK3 $\beta$ were normalized with t-JNK1, t-FAK, t-Src, t-STAT5a and t-GSK3 $\beta$, respectively. Data are presented as mean \pm SEM. $n=5$ per group. ${ }^{*} p<0.05$. 
A

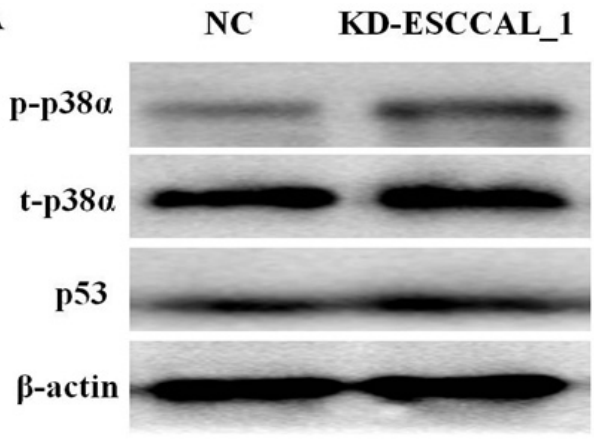

C

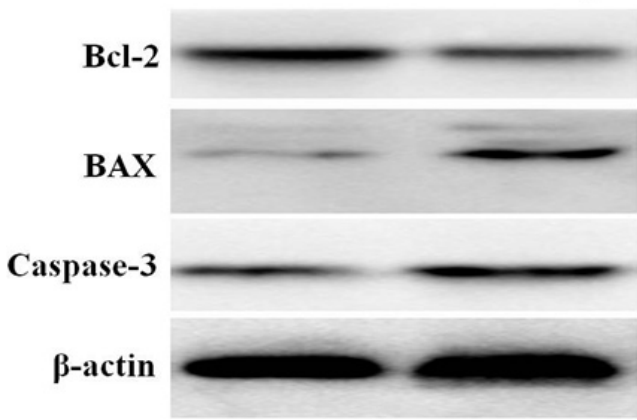

B

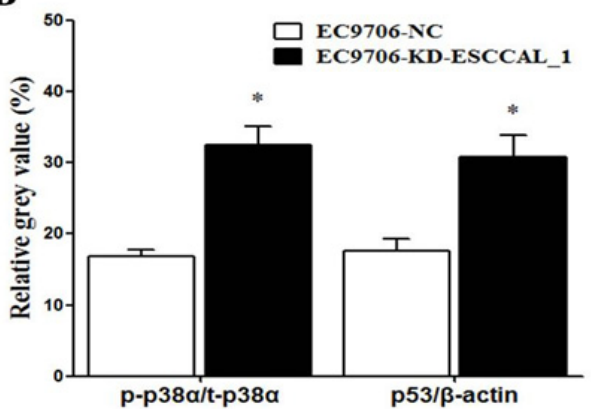

D

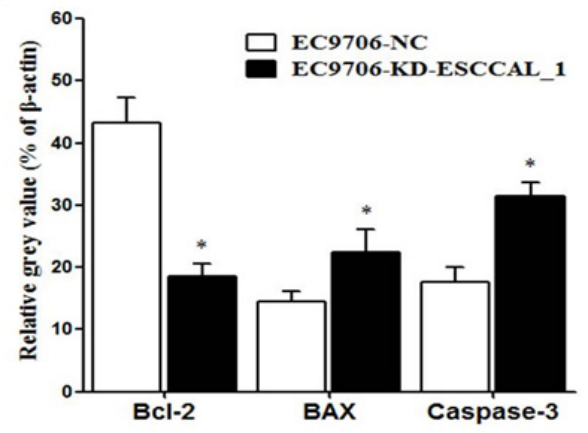

Figure 4: Over-expression of ESCCAL_1 promotes survival and suppresses apoptosis. (A, C) Protein levels of p-p38 $\alpha$, total

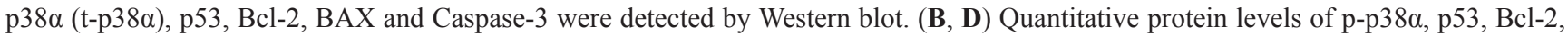
BAX and Caspase- 3 were normalized with t-p38 $\alpha$ or $\beta$-actin. Data are presented as mean \pm SEM. $n=5$ per group. ${ }^{*} p<0.05$.

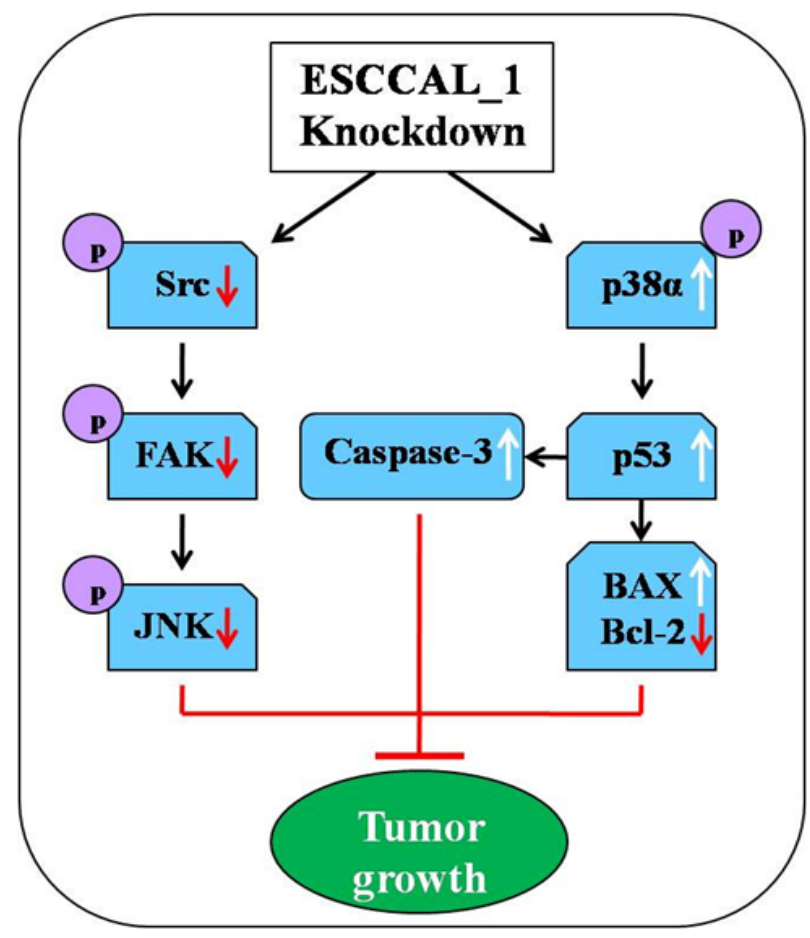

Figure 5: Diagram illustrates the underlying mechanism of ESCCAL_1 knockdown inhibits tumor growth in nude mice. From the phenotype of overexpression of ESCCAL 1 promoting tumor growth to targets screening of phospho-kinase protein array, along with shRNA knockdown of ESCCAL_1 expression, the cross-talk of multiple signaling pathways may participate in ESCCAL_1-mediated oncogenesis. The inactivation of p-Src, p-FAK, p-JNK1, decrease of Bcl-2 expression and increase of p-p38, p53, BAX and Caspase-3 expression in ESCCAL_1 KD group were detected in this study (Red arrows: decrease; white arrows: increase; black arrows: activate/induce). 
that down-regulation of ESCCAL_1 inhibits ESCC tumor growth might be mediated by Src and p38 $\alpha$ pathway.

In summary, our data indicate that knockdown of ESCCAL_1 inhibits tumor growth in an ESCC xenografts mouse model, inactivation of Src and activation of $\mathrm{p} 38 \alpha$ may be two cross-talk pathways responsible to the resulting phynotype. Our study also suggests that ESCCAL_1 is a putative oncogenic lncRNA during tumorigenesis.

\section{MATERIALS AND METHODS}

\section{In vivo tumor growth assay}

Six week old male $\mathrm{BALB} / \mathrm{c}$ nude mice were purchased from the Shanghai Experimental Animal Center, Chinese Academy of Sciences (Shanghai, China). All experimental animal procedures were carried out according to the Ethical Committee of Zhengzhou University. All mice were housed under a $12 \mathrm{~h}$ light/dark cycle and automatically given food and water. In this experiment, the vector hU6-MCS-CMV-EGFP (GV155) (Genechem, China) was used to generate ESCCAL_1shRNA or nonsense-shRNA (under human U6 promoter) according to the manufacture's instruction, the shuttle vector and viral packaging system were cotransfected into HEK293T cells to replicate competent lentivirus [17]. The EC9706 cells infected with ESCCAL_1-shRNA or nonsense-shRNA Lentivirus were used for xenografts as our previously described [17]. Then, the nude mice were divided into two groups: Negative control (NC) $(n=6)$, injection of nonsense-shRNA Lentivirus infected EC9706 cells subcutaneously into the back flank; Knockdown (KD) $(n=6)$, injection of ESCCAL_1-shRNA Lentivirus infected EC9706 cells subcutaneously into the back flank. The tumor volumes were calculated as length $\times$ width $^{2} \times$ 0.5 from day 13 to day 23 every two days, the mice were sacrificed at day 23 after injection and tumor weights were measured for further studies.

\section{Tissue preparation}

All the mice were sacrificed at day 23 after injection and the tumor tissues were immediately harvested. After rinsed in $0.9 \%$ saline solution, the tissues were ground in the liquid nitrogen into a powder separately. Then, the total proteins were extracted from the tumor tissues by using RIPA lysis buffer containing PMSF (RIPA: PMSF = 100:1). Protein concentration of each tissue lysate was quantified by using a NanoDrop 2000 spectrophotometer (Thermo Scientific, USA). The proteins were stored in the refrigerator at $-80^{\circ} \mathrm{C}$ or used for molecular biological analysis directly.

\section{Phospho-kinase antibody array}

The Human Phospho-Kinase Array kit (R\&D Systems, USA) was used to examine the expression of phosphorylated proteins in the tumor tissues according to the manufacturer's instructions. This array specifically determines the relative phosphorylation levels of kinases and proteins associated with cell proliferation and apoptosis. Briefly, after blocking in the array buffer for $1 \mathrm{~h}$ at room temperature, the membranes were incubated with diluted proteins in each well overnight at $4^{\circ} \mathrm{C}$ on a rocking platform shaker. Then, the diluted detection antibody cocktail A or B was added into wells separately for part A or B membranes and incubated for $2 \mathrm{~h}$ at room temperature. After incubating with diluted streptavidinHRP and chemi reagent mix for appropriate time, the immunoreactive dots on the membranes were detected by using the Chemidoc EQ system (BioRad, USA).

\section{Western blot analysis}

Total proteins were denatured at $100^{\circ} \mathrm{C}$ by using a Thermocell cooling \& heating block (Hangzhou Bioer Technology, China). Equal amounts of denatured proteins $(100 \mu \mathrm{g})$ were separated by SDS-PAGE and transferred to a PVDF membrane. After blocking with 5\% (weight: volume) skim milk for $1 \mathrm{~h}$ at room temperature on a rocking platform shaker, the membrane was incubated in the diluted primary antibody overnight at $4^{\circ} \mathrm{C}$. Then, the membrane was incubated with HRP-conjugated secondary antibody for $2 \mathrm{~h}$ at room temperature. Finally, the immunoreactive bands on the membrane were detected by using the Chemidoc EQ system (BioRad, USA). Primary antibodies as follows: anti-STAT5a (1:200, Santa Cruz, USA), anti-p-STAT5a (1:200, Santa Cruz, USA), anti-FAK (1:200, Santa Cruz, USA), anti-p-FAK (1:200, Santa Cruz, USA), anti-GSK-3 $\beta$ (1:200, Boster, China), anti-p-GSK-3 $\beta$ (1:200, Santa Cruz, USA), anti-p38 $\alpha$ (1:200, Santa Cruz, USA), anti-p-p38 $\alpha$ (1:200, Santa Cruz, USA), anti-JNK1 (1:200, Santa Cruz, USA), anti-p-JNK1 (1:200, Boster, China), anti-Src (1:200, Boster, China), anti-p-Src (1:200, Santa Cruz, USA), anti-Caspase-3 (1:200, Santa Cruz, USA), anti-BAX (1:200, Boster, China), anti-Bcl-2 (1:200, Boster, China), anti-p53 (1:200, Boster, China), anti- $\beta$-actin (1:2000, Santa Cruz, USA).

\section{Statistical analysis}

The data were analyzed by using the Graphpad Prism 5 software. All results are presented as Mean \pm Standard error of measurement (SEM). Pairwise comparisons were carried out by using two-tailed student's $t$-test. A value of $P<0.05$ was considered to be statistically significant.

\section{Author contributions}

YBC carried out the experiments and wrote the manuscript. WC and WW designed the experiments, conceived and participated in guiding the experiments and revision of the manuscript. PJL, JYZ and BQB provided 
assistance for Western blot, revision of the manuscript and data analysis separately. All authors read and approved the manuscript for publication.

\section{ACKNOWLEDGMENTS}

We thank all the team members in Dr. Cao's lab for their assistance.

\section{CONFLICTS OF INTEREST}

The authors declared that they have no competing interests.

\section{FUNDING}

This study was funded by the Natural Science Foundation of Henan Province (162300410279), the Science and Technology Program of Henan (152102410088), the major scientific and technological project of Henan (161100311400).

\section{REFERENCES}

1. Wu C, Hu Z, He Z, Jia W, Wang F, Zhou Y, Liu Z, Zhan Q, Liu Y, Yu D, Zhai K, Chang J, Qiao Y, et al. Genomewide association study identifies three new susceptibility loci for esophageal squamous-cell carcinoma in Chinese populations. Nat Genet. 2011; 43:679-84.

2. Torre LA, Bray F, Siegel RL, Ferlay J, Lortet-Tieulent J, Jemal A. Global cancer statistics, 2012. CA Cancer J Clin. 2015; 65:87-108.

3. van Hagen P, Hulshof MC, van Lanschot JJ, Steyerberg EW, van Berge Henegouwen MI, Wijnhoven BP, Richel DJ, Nieuwenhuijzen GA, Hospers GA, Bonenkamp JJ, Cuesta MA, Blaisse RJ, Busch OR, et al, and CROSS Group. Preoperative chemoradiotherapy for esophageal or junctional cancer. N Engl J Med. 2012; 366:2074-84.

4. Chen MQ, Lin QL, Chen YG, Guo JH, Xu BH, Tian Y. Neoadjuvant chemotherapy may not benefit esophageal squamous cell carcinoma patients treated with definitive chemoradiotherapy. J Chin Med Assoc. 2017; 80:636-643.

5. Deng $\mathrm{Y}$, Bian $\mathrm{C}$, Tao H, Zhang H. Improved survival with higher radiation dose for esophageal squamous cell carcinoma patients treated with definitive chemoradiotherapy. Oncotarget. 2017; 8:79662-69. https://doi.org/10.18632/ oncotarget. 19030

6. Chen J, Zhu J, Pan J, Zhu K, Zheng X, Chen M, Wang J, Liao Z. Postoperative radiotherapy improved survival of poor prognostic squamous cell carcinoma esophagus. Ann Thorac Surg. 2010; 90:435-442.

7. He LR, Liu MZ, Li BK, Jia WH, Zhang Y, Liao YJ, Chen YC, Zhang LJ, Guan XY, Zeng YX, Kung HF, Xie D. High expression of EZH2 is associated with tumor aggressiveness and poor prognosis in patients with esophageal squamous cell carcinoma treated with definitive chemoradiotherapy. Int J Cancer. 2010; 127:138-47.

8. Xu F, Zhang J. Long non-coding RNA HOTAIR functions as miRNA sponge to promote the epithelial to mesenchymal transition in esophageal cancer. Biomed Pharmacother. 2017; 90:888-896.

9. Bhan A, Mandal SS. LncRNA HOTAIR: A master regulator of chromatin dynamics and cancer. Biochim Biophys Acta. 2015; 1856:151-64.

10. Liu RT, Cao JL, Yan CQ, Wang Y, An CJ, Lv HT. Effects of LncRNA-HOST2 on cell proliferation, migration, invasion and apoptosis of human hepatocellular carcinoma cell line SMMC-7721. Biosci Rep. 2017; 37:BSR20160532.

11. Wang Y, Kuang H, Xue J, Liao L, Yin F, Zhou X. LncRNA AB073614 regulates proliferation and metastasis of colorectal cancer cells via the PI3K/AKT signaling pathway. Biomed Pharmacother. 2017; 93:1230-1237.

12. Jin J, Chu Z, Ma P, Meng Y, Yang Y. Long non-coding RNA SPRY4-IT1 promotes proliferation and invasion by acting as a ceRNA of miR-101-3p in colorectal cancer cells. Tumour Biol. 2017; 39:1010428317716250.

13. Chen X, Han H, Li Y, Zhang Q, Mo K, Chen S. Upregulation of long noncoding RNA HOTTIP promotes metastasis of esophageal squamous cell carcinoma via induction of EMT. Oncotarget. 2016; 7:84480-85. https:// doi.org/10.18632/oncotarget.12995.

14. Ren K, Li Y, Lu H, Li Z, Li Z, Wu K, Li Z, Han X. Long Non-coding RNA HOTAIR Controls Cell Cycle by Functioning as a Competing Endogenous RNA in Esophageal Squamous Cell Carcinoma. Transl Oncol. 2016; 9:489-497.

15. Luo HL, Huang MD, Guo JN, Fan RH, Xia XT, He JD, Chen XF. AFAP1-AS1 is upregulated and promotes esophageal squamous cell carcinoma cell proliferation and inhibits cell apoptosis. Cancer Med. 2016; 5:2879-2885.

16. Cao W, Wu W, Shi F, Chen X, Wu L, Yang K, Tian F, Zhu M, Chen G, Wang W, Biddle FG, Gu J. Integrated analysis of long noncoding RNA and coding RNA expression in esophageal squamous cell carcinoma. Int J Genomics. 2013; 2013:480534.

17. Hao Y, Wu W, Shi F, Dalmolin RJ, Yan M, Tian F, Chen X, Chen G, Cao W. Prediction of long noncoding RNA functions with co-expression network in esophageal squamous cell carcinoma. BMC Cancer. 2015; 15:168.

18. Pan Z, Mao W, Bao Y, Zhang M, Su X, Xu X. The long noncoding RNA CASC9 regulates migration and invasion in esophageal cancer. Cancer Med. 2016; 5:2442-7.

19. Berndt N, Karim RM, Schönbrunn E. Advances of small molecule targeting of kinases. Curr Opin Chem Biol. 2017; 39:126-132.

20. Shan B, Man H, Liu J, Wang L, Zhu T, Ma M, Xv Z, Chen X, Yang X, Li P. TIM-3 promotes the metastasis of esophageal squamous cell carcinoma by targeting epithelialmesenchymal transition via the Akt/GSK-3 $\beta /$ Snail signaling pathway. Oncol Rep. 2016; 36:1551-61. 
21. Rani A, Murphy JJ. STAT5 in Cancer and Immunity. J Interferon Cytokine Res. 2016; 36:226-37.

22. Yu ZS, Song XH, Gan HM, Geng DZ, Yang J. Activation of Src tyrosine kinase in esophageal carcinoma cells in different regulatory environments and corresponding occurrence mechanism. Genet Mol Res. 2016; 15:15.

23. Lai YH, Chen MH, Lin SY, Lin SY, Wong YH, Yu SL, Chen HW, Yang CH, Chang GC, Chen JJ. Rhodomycin A, a novel Src-targeted compound, can suppress lung cancer cell progression via modulating Src-related pathways. Oncotarget. 2015; 6:26252-65. https://doi.org/10.18632/ oncotarget. 4761 .

24. Wheeler DL, Iida M, Dunn EF. The role of Src in solid tumors. Oncologist. 2009; 14:667-78.

25. Hui L, Bakiri L, Stepniak E, Wagner EF. p38alpha: a suppressor of cell proliferation and tumorigenesis. Cell Cycle. 2007; 6:2429-33.

26. Zheng ST, Zhang CS, Qin X, Gen YH, Liu T, Sheyhidin I, Lu XM. The status of phosphorylated p38 in esophageal squamous cell carcinoma. Mol Biol Rep. 2012; 39:5315-21.
27. Zheng F, Tang Q, Wu J, Zhao S, Liang Z, Li L, Wu W, Hann S. p38 $\alpha$ MAPK-mediated induction and interaction of FOXO3a and p53 contribute to the inhibited-growth and induced-apoptosis of human lung adenocarcinoma cells by berberine. J Exp Clin Cancer Res. 2014; 33:36.

28. Yabasin IB, Lu Z, Yu JC, Wen Q. Cisatracurium-induced proliferation impairment and death of colorectal cancer cells, HCT116 is mediated by p53 dependent intrinsic apoptotic pathway in vitro. Biomed Pharmacother. 2017; 91:320-329.

29. Lv J, Lin S, Peng P, Cai C, Deng J, Wang M, Li X, Lin R, Lin Y, Fang A, Li Q. Arenobufagin activates p53 to trigger esophageal squamous cell carcinoma cell apoptosis in vitro and in vivo. Onco Targets Ther. 2017; 10:1261-1267.

30. Moghtaderi H, Sepehri H, Attari F. Combination of arabinogalactan and curcumin induces apoptosis in breast cancer cells in vitro and inhibits tumor growth via overexpression of p53 level in vivo. Biomed Pharmacother. 2017; 88:582-594. 